\title{
The Phase-1 Upgrade of the ATLAS Level-1 Endcap Muon Trigger
}

\author{
Shunichi Akatsuka, on behalf of the ATLAS Collaboration \\ Kyoto University
}

\begin{abstract}
The Level-1 muon trigger for the ATLAS experiment identifies muons with high transverse momentum. Under the high luminosity condition in LHC Run 3, more powerful trigger system is needed to reject the backgrounds. New trigger processor board for Run 3 has been produced to achieve the required performance, by combining information from five different detectors. The performance of the new board and the readout system has been confirmed by a beam test. The new algorithms to make use of the new detectors have been developed, and are tested by MC simulation. With the new algorithms, the trigger rate is estimated to become lower than the required rate in Run 3.
\end{abstract}

\section{Introduction}

LHC Run 3 is planned to start in 2021, with an instantaneous luminosity of $3.0 \times 10^{34} \mathrm{~cm}^{-2} \mathrm{~s}^{-1}$, which is twice as much as the luminosity in the current run (Run 2). Despite the higher event rate, the data recording rate will not increase significantly. In this situation, the requirements on the trigger system will be more severe. The ATLAS detector [I] needs an upgrade before LHC Run 3 , to enhance its performance to cope with these high luminosity conditions (collectively this effort is known as the Phase-1 Upgrade).

The Level-1 trigger is at the first level in the ATLAS trigger system. It consists of dedicated trigger processor hardware, performing fast selection for all the collision events at $40 \mathrm{MHz}$ to reduce the event rate down to $100 \mathrm{kHz}$ within a fixed latency of $\sim 2.5 \mu$ s. This paper focuses on the Phase-1 Upgrade of the Level-1 muon endcap trigger system.

\section{Phase-1 Upgrade of the Level-1 Muon Trigger}

The total Level-1 trigger rate assigned for muons in Run 3 has been defined to be $19 \mathrm{kHz}$, considering other triggers and physics requirements [2]. A large fraction of the muon trigger rate will be occupied by the lowest-threshold unprescaled Level-1 single muon trigger (primary muon trigger). In Run 3, the primary muon trigger should have a transverse momentum $\left(p_{\mathrm{T}}\right)$ threshold of $20 \mathrm{GeV}$ and a rate below $15 \mathrm{kHz}$ at Run 3 peak luminosity, as well as high trigger efficiency for muons with $p_{\mathrm{T}}$ over the threshold. Without an upgrade of the trigger system, the rate for this trigger is expected to exceed $28 \mathrm{kHz}$. Thus a 
more powerful trigger strategy is needed to achieve a $\sim 50 \%$ rate reduction while keeping the trigger threshold and efficiency.

From previous studies on Run 1 muon trigger performance, it is known that more than $90 \%$ of the muon trigger candidates in the endcap region are due to background events $[Z]$. The major part of these background triggers are due to events with no associated reconstructed muons. These background triggers are known as "fake" triggers, caused by charged particles emerging from the beam pipe. Other background triggers are due to muons with $p_{\mathrm{T}}$ below the threshold. The main strategy of the upgrade is to eliminate the fake triggers and the low- $p_{\mathrm{T}}$ muons by implementing a powerful trigger algorithm using several new detectors introduced in Run 3.

The detectors for the endcap muon trigger in Run 3 are shown in Fig. ஐ. The toroidal magnetic field bends the muon tracks, so that the $p_{\mathrm{T}}$ can be calculated from the track angles in the Thin-Gap Chambers (TGCs) [3] installed outside

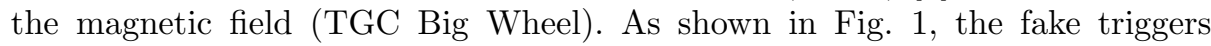
create muon-like tracks in the TGCs, but do not correspond with any hits in the detectors inside the field. Thus the fake triggers can be eliminated by requiring a coincidence between hits in the TGC Big Wheel and the detectors inside the field. The largest area in the endcap region is covered by the New Small Wheel (NSW) [4], which has high position- and angle resolution. By utilising its information with high resolution, low- $p_{\mathrm{T}}$ muon candidates can also be rejected highly effectively.

\section{Development of the Trigger Processor/Readout System}

A new trigger processor board, called Sector Logic (SL) has been developed to combine hit information from the detectors shown in Fig. $\mathbb{W}$. A Xilinx Kintex-7 $\mathrm{XC7K325T}$ has been chosen as the main processor FPGA, which has 16 multigigabit GTX transceivers [6]. The new SL has 12 optical inputs and outputs for the GTX transceivers, each operated at a transfer rate of 6.4 Gbps. These I/Os for the GTX add up to 76.8 Gbps input data rate in total, which is fast enough to receive information from the NSW and other detectors inside the magnetic field. The new SL also has 14 input ports for G-Link connections, which are mainly used to receive information from the TGCs.

The trigger decision information, as well as the raw information received by the SL, are read out to debug and improve the trigger algorithm. The SROD, a software-readout processor implemented on a commercial PC, collects data with TCP/IP from 12 new SL boards. In the new system, another new board called a TTC Fan-out provides the event ID information to the 12 SLs and the SROD. The SROD checks the event IDs in the data received from the SLs, packs them into a certain format, then sends them to the ATLAS Readout System (ROS). A new PCI-express card has also been produced to send data to the ROS via the S-LINK [7] protocol. An integration test of the readout system has been done using the TGC detectors and the muon beam at the SPS beam facility. Stable readout and data recording have been successfully demonstrated. 


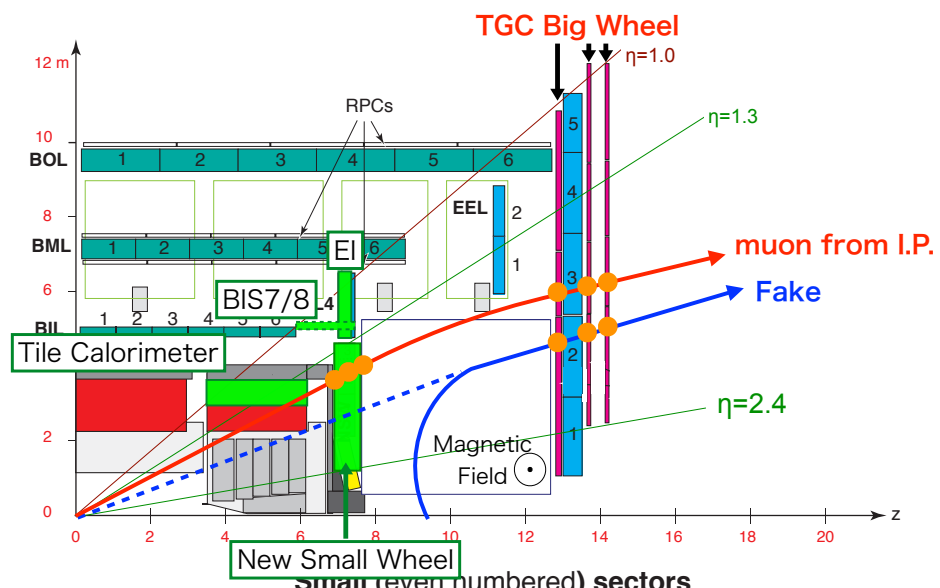

Fig. 1. ${ }^{1}$ The ATLAS detector in the $y-z$ plane. The interaction point (I.P.) is at the origin, and the beam pipe corresponds to the $z$-axis. The four detectors that can be used for coincidence triggering with the TGC Big Wheel are shown by the green boxes: TGC EI [3], new RPC [3] (BIS7/8), Tile Calorimeter [5] and the New Small Wheel. The red line shows a track made by a muon from the I.P., and the blue line shows a track by a fake. The fake tracks do not make coincide with hits in the detectors inside the magnetic field.

\section{Development of the trigger algorithm}

Two types of matching algorithm have been considered; the position matching algorithm and the angle matching algorithm. The position matching algorithm requires hits in both TGC and NSW to reject the fake triggers. With significantly high position resolution by NSW $(\sim 0.05$ in $\eta)$, low- $p_{\mathrm{T}}$ candidates can also be rejected. The angle matching algorithm makes use of the high angular resolution of NSW ( $\sim 1 \mathrm{mrad}$.). This information can be used to estimate the angles inside the magnetic field more accurately, which leads to a better $p_{\mathrm{T}}$ resolution.

The performance of the trigger logic has been tested using MC simulation samples, and is validated with the data taken in 2016 with $\sqrt{s}=13 \mathrm{TeV}$. When both of the position and angle matching algorithms are applied, the low- $p_{\mathrm{T}}$ candidates are rejected to a large degree while keeping the efficiency for the events with muons with $p_{\mathrm{T}}>20 \mathrm{GeV}$ (Fig. $\mathbf{Z}$ ). Note that the fake triggers are not included in Fig. Q, as they do not have associated muon candidates and so the $p_{\mathrm{T}}$ cannot be defined. Together with the study on fake trigger rejection in the TDAQ TDR [2], the trigger rate for muons with $p_{\mathrm{T}}>20 \mathrm{GeV}$ at the luminosity of $3.0 \times 10^{34} \mathrm{~cm}^{-2} \mathrm{~s}^{-1}$ is estimated to become smaller than $13 \mathrm{kHz}$, which meets the Run 3 requirement of $15 \mathrm{kHz}$.

\footnotetext{
${ }^{1}$ Copyright 2017 CERN for the benefit of the ATLAS Collaboration. CC-BY-4.0 license
} 


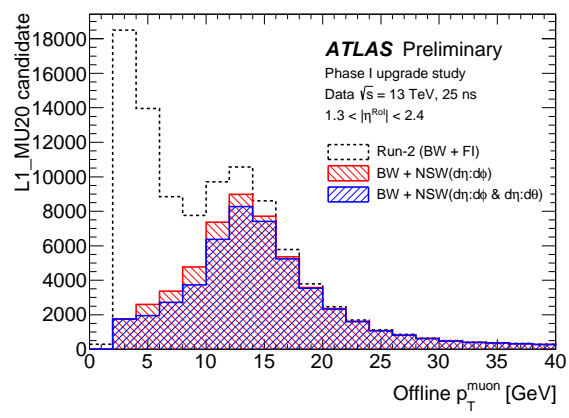

Fig. 2. ${ }^{1} p_{\mathrm{T}}$ distribution of the muons that passed the Level-1 muon trigger [ 8$]$. The dashed line shows the distribution of the Level-1 trigger candidate muons in the Run 2 trigger system. The red (blue) histogram shows the distribution after the position (position + angle) matching algorithm. Low- $p_{\mathrm{T}}$ candidates are rejected effectively, while keeping $96 \%$ efficiency for events associated with muons with $p_{\mathrm{T}}>20 \mathrm{GeV}$.

\section{Conclusion}

A new trigger processor board for the Level-1 endcap muon trigger in Run 3 has been produced to combine information from five different detectors. Together with the new readout system, the performance of the board has been verified by a beam test. New trigger algorithms for Run 3 have been developed: the position matching and the angle matching algorithms. By applying both of the new algorithms, the trigger rate of the primary muon trigger will be $13 \mathrm{kHz}$ at Run-3 peak luminosity, which meets the ATLAS trigger requirement.

\section{References}

1. ATLAS Collaboration, "The ATLAS Experiment at the CERN Large Hadron Collider", JINST 3 (2008) S08003.

2. ATLAS Collaboration, "Technical Design Report for the Phase-I Upgrade of the ATLAS TDAQ System", CERN-LHCC-2013-018.

3. ATLAS Collaboration, "ATLAS muon spectrometer: Technical Design Report", CERN-LHCC-97-022.

4. T.Kawamoto et al, "New Small Wheel Technical Design Report", CERN-LHCC2013-006.

5. ATLAS Collaboration, "ATLAS tile calorimeter : Technical Design Report", CERNLHCC-96-042.

6. Xilinx Inc., "7 Series FPGAs GTX/GTH Transceivers User Guide", https://www.xilinx.com/support/documentation/user_guides/ug476_7Series_ Transceivers.pdf.

7. H. C. van der Bij, et al., "S-LINK, a Data Link Interface Specification for the LHC Era", IEEE Trans. Nucl. Sci. 44 (1997) 398.

8. ATLAS Collaboration, "L1 Muon Trigger Public Results", https://twiki.cern. ch/twiki/bin/view/AtlasPublic/L1MuonTriggerPublicResults. 